\title{
Treatment outcomes of differentiated thyroid cancer with distant metastasis improve by tyrosine kinase inhibitors
}

\author{
HIROYUKI IWASAKI $^{1}$, HARUHIKO YAMAZAKI ${ }^{1}$, HIROTAKA TAKASAKI $^{2}$, NOBUYASU SUGANUMA ${ }^{1}$, \\ RIKA SAKAI $^{2}$, HIROTAKA NAKAYAMA ${ }^{3}$, SHINSUKE HATORI ${ }^{4}$, SOJI TODA $^{5}$ and KATSUHIKO MASUDO ${ }^{5}$ \\ Departments of ${ }^{1}$ Breast and Endocrine Surgery and ${ }^{2}$ Oncology, Kanagawa Cancer Center, Asahi-ku, Yokohama, \\ Kanagawa 241-8515; ${ }^{3}$ Department of Surgical Treatment, Yokohama City University Hospital, Kanazawa-ku, \\ Yokohama, Kanagawa 236-0004; ${ }^{4}$ Department of Surgery, Hiratsuka Kyosai Hospital, \\ Hiratsuka, Kanagawa 254-8502; ${ }^{5}$ Department of Breast and Thyroid Surgery, \\ Yokohama City University Medical Center, Yokohama, Kanagawa 232-0024, Japan
}

Received October 15, 2018; Accepted February 28, 2019

DOI: $10.3892 / \mathrm{ol} .2019 .10180$

\begin{abstract}
In patients with distant metastasis, treatment for differentiated thyroid cancer (DTC) includes complete total thyroidectomy, followed by radioactive iodine (RAI) therapy for metastatic lesions. Tyrosine kinase inhibitor (TKI) treatment is the final treatment option for metastatic lesions, which is incurable with surgery/RAI therapy. The present study examined whether treatment outcomes for DTC in patients with distant metastasis improved following TKI treatment. This study included 147 patients (median age, 71; range, 33-91 years) who underwent surgery in our hospitals and were diagnosed with distant metastasis. Disease progression was observed in 70 patients, of whom 56 were treated with TKI (TKI group); 14 refused TKI treatment or showed no treatment indication [untreated (UT) group]. Disease progression and treatment outcomes were assessed using imaging evaluations. The present study investigated thyroglobulin doubling time (Tg-DT) and Tg antibody presence/absence and their relation to disease progression. Overall survival following disease progression between the two groups was compared. The study included 22 cases of sorafenib, 49 of lenvatinib,
\end{abstract}

Correspondence to: Dr Hiroyuki Iwasaki, Department of Breast and Endocrine Surgery, Kanagawa Cancer Center, 2-3-2 Nakao Asahi-ku, Yokohama, Kanagawa 241-8515, Japan

E-mail: iwasaki.h@kcch.jp

Abbreviations: DTC, differentiated thyroid cancer; RAI, radioactive iodine; AE, adverse event; TKI, tyrosine kinase inhibitor; UT, untreated; Tg-DT, thyroglobulin doubling time; PR, partial response; SD, stable disease; PD, progressive disease; RECIST, response evaluation criteria in solid tumors; $\mathrm{Tg}$, thyroglobulin; $\mathrm{TgAb}$, thyroglobulin antibody; CR, complete remission; TTF, time to treatment failure; OS, overall survival; DCR, disease control rate; CT, computed tomography

Key words: differentiated thyroid cancer, tyrosine kinase inhibitors, overall survival, retrospective study, thyroglobulin doubling time and 15 involving TKIs. The mean dosing period for sorafenib was 153 days and for lenvatinib was 462 days. In the TKI group, 16, 26, and 9 patients exhibited partial responses (PRs), stable disease (SD), and progressive disease (PD), respectively, whereas 5 patients were not evaluable. The disease control rate $(\mathrm{DCR})(\mathrm{PR}+\mathrm{SD})$ was $75.0 \%$. A total of 16 patients died in the TKI group, whereas 10/14 patients in the UT group died. Survival curves for the groups were significantly different. TKI treatment improved the prognosis of patients with distant metastasis and PD.

\section{Introduction}

Differentiated thyroid cancer (DTC) has a relatively good prognosis, but distant metastasis can lead to death due to progressive disease (PD) (1). The primary treatment strategy for patients with DTC and distant metastasis is total thyroidectomy and regional lymph node dissection, followed by I-131 radioactive iodine (RAI) therapy. Patients that develop distant metastasis after hemi-thyroidectomy typically undergo total thyroidectomy followed by RAI therapy. Other standard treatments include surgery, RAI therapy (2), and thyroid hormone suppression therapy (1). External irradiation can be performed to control pain due to bone metastases or local recurrence lesions. Long-term survival with remnant tumors is possible if local disease control is good; the 10-year survival rate for patients with distant metastatic thyroid cancer ranges from 26-60\% (3-5). However, activities of daily living may decline due to bone or pulmonary metastasis.

According to the American Thyroid Association (6) and the Union for International Cancer Control guidelines (7), tyrosine kinase inhibitor (TKI) treatment is recommended for progressive RAI-refractory DTC. The TKIs sorafenib (8) and lenvatinib (9) were previously described for treating RAI-refractory DTC following disease progression. Both lenvatinib and sorafenib have been reported to target RAI refractory metastatic lesions after total thyroidectomy. For lenvatinib, there was at least one measurable lesion without iodine uptake on any iodine-131 scan and at least one measurable lesion that had progressed (10). For sorafenib, there was at 
least one target lesion without iodine uptake (11). To the best of our knowledge, no study has investigated the frequency of disease progression in distant metastasis from DTC nor has anyone reported on patient prognosis in those with PD that were treated with sorafenib and/or lenvatinib or not treated with TKI. Sorafenib has been approved for the treatment of RAI-refractory and progressive DTC in Japan since June 2014, with lenvatinib being cleared the following year (12). In this study, we retrospectively investigated whether the use of TKIs improved the long-term treatment outcomes of distant metastasis from DTC. The aims of this study were to clarify the factors associated with prognosis among patients with DTC with distant metastasis and evaluate TKI drugs in clinical practice.

\section{Materials and methods}

Patients. The current study included 147 patients (59 men, 88 women; median age, 71; range, 33-91 years) diagnosed with distant metastasis from DTC who were treated in our hospitals between April 2015 and August 2018. Informed consent was obtained from all individual participants included in the study. Cancer staging was determined according to the cancer eighth edition cancer staging manual by the American joint committee (13). DTC patients already diagnosed with distant metastasis in stage IV-B (age >55) and in stage II (age <55), follow-up cases, and those diagnosed with other stage initially and recognized distant metastasis later were included. Patients not continuing treatment at our hospitals were excluded. Pathological diagnoses included papillary carcinoma in 122 patients and follicular carcinoma in 25. Patients with medullary carcinoma were excluded due to varying treatment strategy and tumor markers. Overall, 77 patients $(52.4 \%)$ exhibiting stable disease (SD) were followed up in our outpatient clinics (SD group). These patients underwent total thyroidectomy as the initial surgery, or completion surgery was performed when distant metastases were recognized, with all patients undergoing at least one round of RAI therapy. Denosumab therapy and external irradiation were performed to relieve pain caused by bone metastasis and to prevent osteoporosis (14), and $120 \mathrm{mg}$ denosumab was administered subcutaneously at least once every 2 months. Decompression and volume reduction surgery in addition to external irradiation were performed to prevent paralysis in cases of vertebral bone metastasis. Of the 147 total cases, disease progression was observed in 70 (47.6\%), 56 of which were treated with TKI (TKI group). Disease progression was diagnosed as an increase of $\geq 20 \%$ based on the response evaluation criteria in solid tumors (RECIST) (version 1.1) criteria (15) or the appearance of a new lesion on imaging within 1 year. Of the 14 patients $(9.5 \%)$ who were not treated with TKIs (untreated [UT] group), three refused treatment and nine were excluded because they exhibited no treatment indications for TKI or other treatments due to a wide range of tracheal invasion, common carotid infiltration, and high risk of bleeding. Patient subgroups were listed in Fig. 1. Numbers and parameters of the patient groups are listed in Table I.

TKI treatment. Sorafenib and lenvatinib were administered to 22 and 49 patients, respectively. Among patients who were administered both drugs, the initial treatment was sorafenib in 12 patients and lenvatinib in three. Because only sorafenib could be used until June 2015, we decided to use it as the first-choice TKI. After June 2015, lenvatinib, which has greater efficacy, was selected. The maximum size of the evaluated lesion, pathological diagnosis, thyroglobulin $(\mathrm{Tg})$ level, and Tg doubling time (Tg-DT) (16) were compared between the $\mathrm{SD}, \mathrm{UT}$, and TKI groups. In the course of treatment, transient $\mathrm{Tg}$ oscillations represent a frequent phenomenon that may not necessarily reflect morphologic tumor progression (17). A Tg level of $<1 \mathrm{ng} / \mathrm{ml}$ during SD indicated a lack of disease progression and rendered it impossible to calculate Tg-DT. That is, when calculated with a trace amount $\mathrm{Tg}$ of $\leq 1 \mathrm{ng} / \mathrm{ml}$, the calculated Tg-DT becomes a strange numerical value, and it cannot be evaluated. Meanwhile, decreases in Tg levels, which are calculated as negative Tg-DT values, denoted successful treatment using RAI therapy and/or external irradiation. Tg antibody (TgAb) negativity was determined as previously described (16). Tg levels before and after RAI therapy were excluded from the calculation due to the effect of discontinuing thyroid hormone therapy. We also excluded patients failing to comply with daily thyroid hormone use and those with elevated thyroid-stimulating hormone levels. Patients for whom Tg-DT could be calculated in the SD and TKI groups are described in the scatter plot (Fig. 2). In addition, we determined the treatment outcomes for each target lesion, as the timing of TKI treatment depends on the metastatic lesion. Changes in tumor diameter before and after TKI treatment are illustrated for each target lesion (Fig. 3A-C). The radiologic response to TKI therapy was classified according to RECIST version 1.1 criteria as complete remission (CR), partial response (PR), SD, or PD. Safety was assessed based on the Common Terminology Criteria for Adverse Events version 3.0. The disease control rate (DCR) was defined as the percentage of patients with $\mathrm{CR}$, PR, and SD. Patients treated with sorafenib and those treated with lenvatinib were separately examined to determine the respective treatment efficacy; the administration period for cases in which treatment was discontinued is graphically shown as the time to treatment failure (TTF) together with the reason for discontinuation. The treatment period is also graphically shown for each ongoing treatment case, and the treatment effect is described (Fig. 4A and B).

Evaluation. Next, we investigated whether TKI treatment improved the treatment outcomes of distant metastasis from DTC by comparing the overall survival (OS) between the UT and TKI groups (Fig. 5A). We compared the OS by target lesions, pulmonary metastasis, bone metastasis, and local recurrence (Fig. 5B). OS was defined as the time between the date of initial diagnosis or the initial surgery in the UT group, the date of the initial surgery in the TKI group, and the date of death or final evaluation.

Statistical analysis. The OS was calculated using the Kaplan-Meier method on the SPSS software. Kaplan-Meier estimator on the SPSS software (version 24; IBM Corp., Armonk, NY, USA) was used to calculate the OS, and log-rank and Bonferroni tests were applied. $\mathrm{P}<0.05$ was considered to indicate a statistically significant difference. The OS was not calculated in the SD group because all of those patients 
Table I. Patient characteristics.

A, Factor

\begin{tabular}{lccc}
\hline & & Subgroup & \\
Group & SD & TKI & UT \\
\hline $\mathrm{n}$ & 77 & 56 & 14 \\
Age & $71.00(33.00,91.00)$ & $70.00(41.00,84.00)$ & $70.00(47.00,84.00)$ \\
Sex $(\%)$ & & & 0.983 \\
Female & $45(58.4)$ & $35(62.5)$ & $8(57.1)$ \\
Male & $32(41.6)$ & $21(37.5)$ & $6(42.9)$ \\
Pathology $(\%)$ & $12(15.6)$ & $11(19.6)$ & $2(14.3)$ \\
FTC & $65(84.4)$ & $45(80.4)$ & $12(85.7)$ \\
PTC & & & 0.744 \\
PS $(\%)$ & $66(85.7)$ & $25(44.6)$ & $4(28.6)$ \\
0 & $11(14.3)$ & $25(44.6)$ & $5(35.7)$ \\
1 & $0(0.0)$ & $6(10.7)$ & $5(35.7)$ \\
\hline
\end{tabular}

B, Characteristics

\begin{tabular}{|c|c|c|c|c|}
\hline \multirow[b]{2}{*}{ Group } & \multicolumn{3}{|c|}{ Subgroup } & \multirow[b]{2}{*}{ P-value } \\
\hline & SD & TKI & UT & \\
\hline Pulmonary metastasis (\%) & $58(75.3)$ & $47(83.9)$ & $11(78.6)$ & 0.486 \\
\hline Bone metastasis $(\%)$ & $12(15.6)$ & $22(40.0)$ & $5(35.7)$ & $0.005^{\mathrm{a}}$ \\
\hline $\mathrm{LN}$ recurrence $(\%)$ & $12(15.8)$ & $31(55.4)$ & $7(70.0)$ & $<0.001^{\mathrm{a}}$ \\
\hline Local recurrence $(\%)$ & $12(16.0)$ & $15(27.3)$ & $8(72.7)$ & $<0.001^{\mathrm{a}}$ \\
\hline Died $(\%)$ & $1 \quad(1.3)$ & $16(29.1)$ & $10(71.4)$ & $<0.001^{\mathrm{a}}$ \\
\hline Diameter & $7.00(3.00,76.00)$ & $25.00(9.00,99.00)$ & $30.00(3.00,60.00)$ & $<0.001^{\mathrm{a}}$ \\
\hline Follow up & $8.78(0.13,31.20)$ & $9.93(2.12,30.53)$ & $5.38(0.52,14.19)$ & $0.032^{\mathrm{a}}$ \\
\hline Tg-DT & $2.23(0.14,9.69)$ & $1.53(0.31,7.29)$ & $1.20(0.38,2.51)$ & 0.182 \\
\hline $\operatorname{TgAb}(\%)$ & $9(11.7)$ & $17(32.1)$ & $4(28.6)$ & $0.014^{\mathrm{a}}$ \\
\hline
\end{tabular}

SD, stable disease; TKI, tyrosine kinase inhibitor; UT, untreated patients; PS, performance status; $\mathrm{TgAb}^{+}$, thyroglobulin antibody positivity;

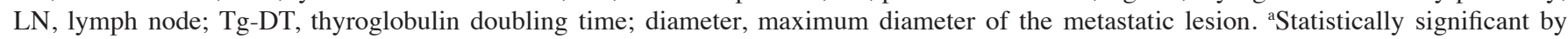
Kruskal-Wallis test.

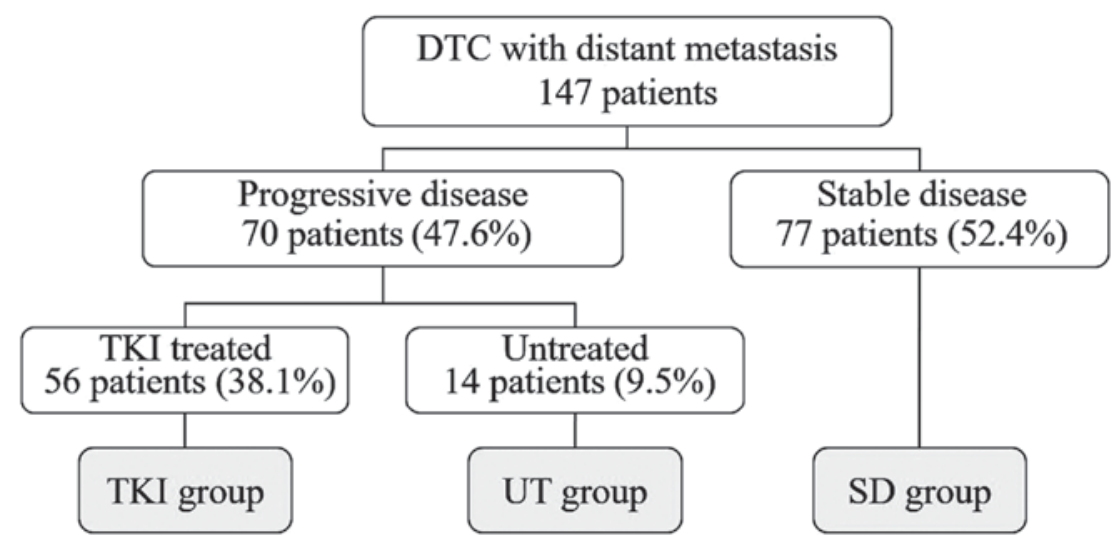

Figure 1. Subgroups in 147 DTC patients with distant metastasis. Of the 147 patients, 77 patients had progressive disease, 56 of whom had TKI treatment (TKI group), 14 who had followed treatment without treatment (UT group), 70 who did not demonstrate disease progression (SD group). DTC, differentiated thyroid cancer; TKI, tyrosine kinase inhibitor; UT, untreated; SD, stable disease. 
Table II. Antitumor efficacy of TKIs.

\begin{tabular}{|c|c|c|c|c|}
\hline \multirow[b]{2}{*}{ Characteristics } & \multicolumn{4}{|c|}{ Metastatic sites } \\
\hline & Total & Pulmonary & Bone & Others \\
\hline $\mathrm{N}$ & 56 & 23 & 9 & 24 \\
\hline Tumor size prior to TKI (mm) & $24.0(9.99)$ & $17.0(12.44)$ & $50.0(20.99)^{\mathrm{a}}$ & $27.5(9.55)$ \\
\hline Complete response & 0 & 0 & 0 & 0 \\
\hline Partial response & 16 & 9 & 0 & 7 \\
\hline Stable disease & 26 & 8 & 7 & 11 \\
\hline Progressive disease & 9 & 4 & 1 & 4 \\
\hline Not evaluable & 5 & 2 & 1 & 2 \\
\hline Response rate $(\%)$ & 28.5 & 39.1 & 0.0 & 29.2 \\
\hline Disease control rate $(\%)$ & 75.0 & 73.9 & 77.8 & 75.0 \\
\hline
\end{tabular}

TKI, tyrosine kinase inhibitor. ${ }^{a}$ The tumor size was significantly larger than the other 2 groups. $\mathrm{P}=0.77 \times 10^{-6}$, $(\mathrm{P}<0.001)$ by Kruskal-Wallis test.

survived, except one who died from other disease. The OS of the TKI and UT group were validated by log-rank test only. Comparison of the median values among three groups was done using the Kruskal-Wallis test and the statistically significant difference was set at $\mathrm{P}<0.05$. These statistical analyses were performed using EZR (Saitama Medical Center, Jichi Medical University, Saitama, Japan), a graphical user interface for R (The R Foundation for Statistical Computing, Vienna, Austria); more precisely, it is a modified version of $\mathrm{R}$ commander designed to add statistical functions frequently used in biostatistics (18).

\section{Results}

Patients. Patient characteristics and parameters are shown in Table I. The median maximum diameter of the metastatic lesion in the SD group was $7 \mathrm{~mm}$, which was statistically smaller $(\mathrm{P}<0.001)$ than in the TKI group $(25 \mathrm{~mm})$ and in the UT group $(30 \mathrm{~mm})$. Performance status was better in the SD group, which also had a lower rate of bone metastasis and recurrence of regional node metastasis. Meanwhile, other parameters, such as age, sex, histology etc. were similar between the groups. Treatment outcomes are shown in Table II. In the TKI group, $16(28.6 \%), 26$ (46.4\%), and nine patients (16.1\%) exhibited PR, $\mathrm{SD}$, and PD, respectively, whereas five patients (8.9\%) were not evaluable, thus the DCR was $75.0 \%$ (42 of 56). Sixteen patients $(29.1 \%)$ in the TKI group died, with causes of death being adverse events (AEs) in three (two grade 5 bleeding cases and one gastrointestinal perforation case), disease progression following treatment discontinuation in eight, anaplastic transformation in two, and aspiration pneumonitis and other complications in three. Meanwhile, one patient in the SD group died from other disease, whereas 10 of $14(71.4 \%)$ in the UT group died. The median observation periods were 8.78 (range, 0.13-31.20) years for the SD group, 9.93 (range, 2.12-30.53) years for the TKI group, and 5.38 (range, 0.52-14.19) years for the UT group.

$\operatorname{TgAb}$ and $\mathrm{Tg}-\mathrm{DT}$. TgAb positivity and $\mathrm{Tg}$ level were not informative in nine of 77 patients $(11.7 \%)$ in the SD group,

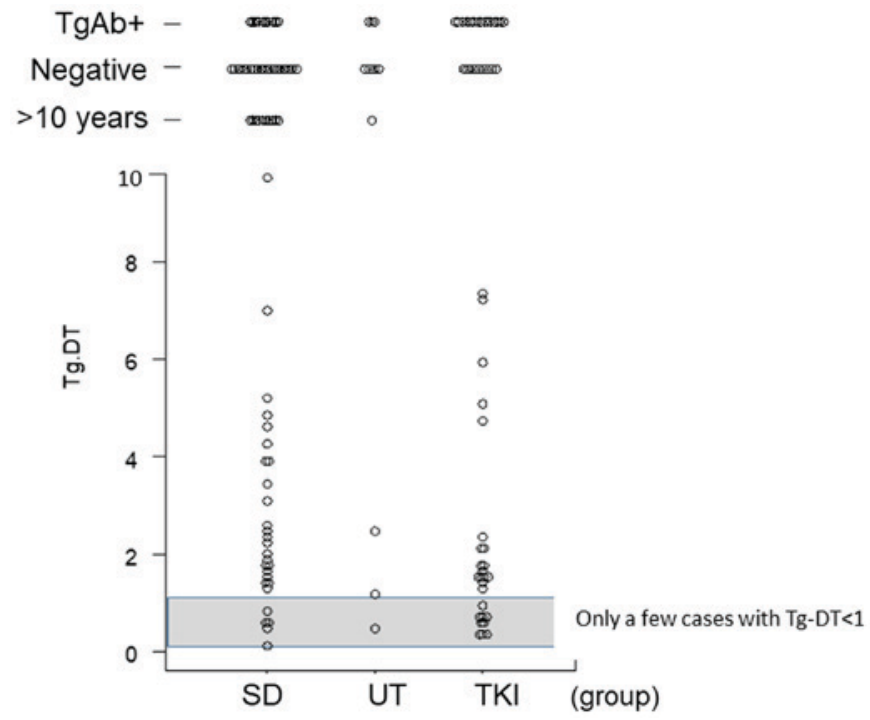

Figure 2. Thyroglobulin doubling time (Tg-DT) distribution in each group This plot graph demonstrates Tg-DT distributions in patients in the UT, TKI, and SD groups. The vertical axis shows doubling time (year). In the top graph, non-informative cases are listed; patients with $\mathrm{TgAb}$ positive $\mathrm{Tg}$-DT were calculated as a negative number, and Tg-DT was $>10$ years. The gray zone demonstrates the area of Tg-DT were within one year, which was a potential indicator for rapid progression. SD, stable disease; UT, untreated; TKI, tyrosine kinase inhibitor.

17 of $56(32.1 \%)$ in the TKI group, and three of $14(21.4 \%)$ in the UT group, and these rates were statistically significant $(\mathrm{P}=0.014)$. The $\mathrm{Tg} \mathrm{Ab}$-positive rate increased with disease progression. The median Tg-DTs were 2.23 (range, 0.14-9.69), 1.53 (range, 0.31-7.29), and 1.20 (range, 0.38-2.51) in the SD, TKI, and UT groups, respectively, and these rates were not statistically significant $(\mathrm{P}=0.18)$. If Tg-DT is measured for a value of 10 years or more, or it becomes a negative value, it can be concluded that there is no disease progression. The number of patients with such values was 36 of $77(41.6 \%)$ in the SD group, 9 of $56(16.1 \%)$ in the TKI group, and 9 of $14(64.3 \%)$ in the UT group. The total number of informative cases was 32 of 77 (41.6\%), 30 of $56(53.6 \%)$, and 2 of 


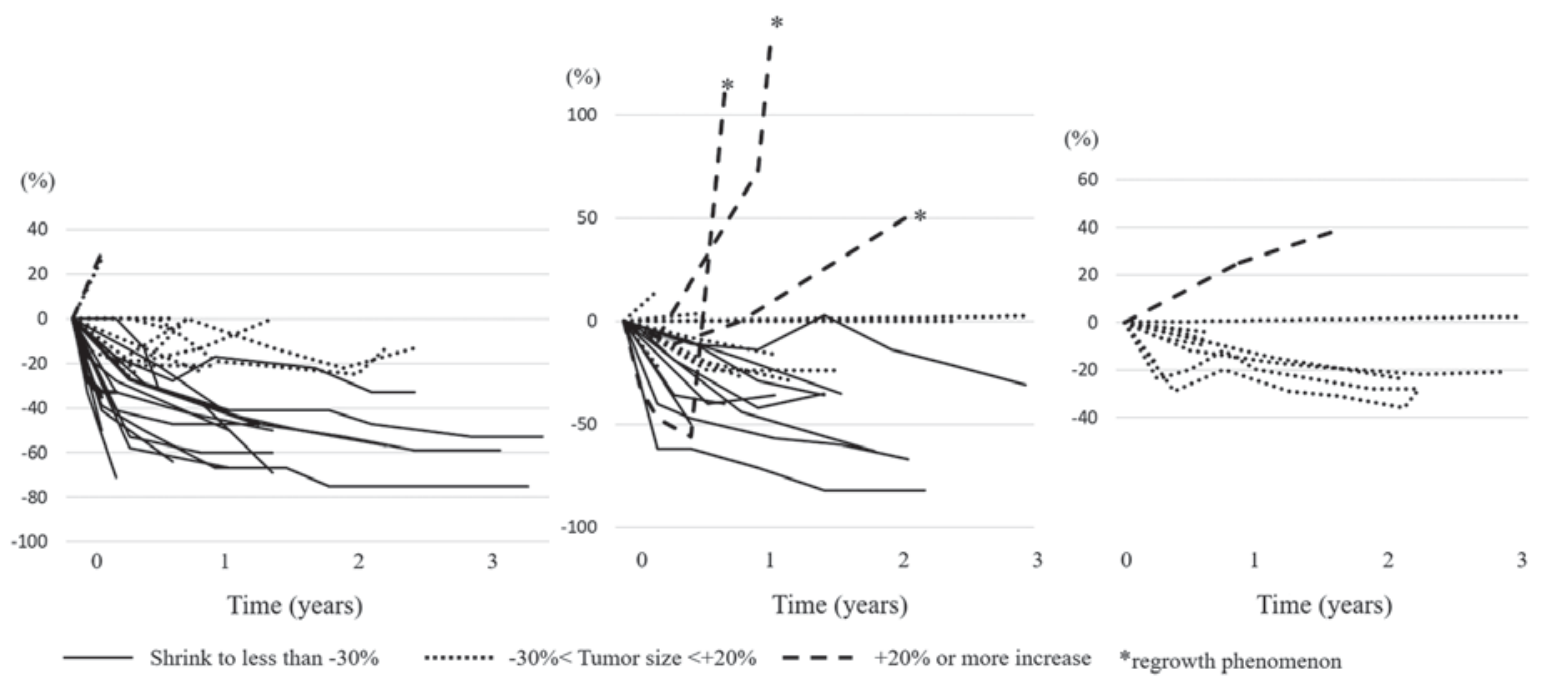

Figure 3. (A) Tumor changes (increase or decrease) compared with baseline after TKI treatment for pulmonary metastatic lesion cases. Only two patients showed tumor growth and demonstrated treatment failure. (B) Tumor changes (increase or decrease) compared with baseline after TKI treatment in unresectable local recurrence or nodal metastasis cases. Asterisks ndicate regrowth phenomenon in patients following treatment failure. (C) Tumor changes (increase or decrease) compared with baseline after TKI treatment in bone metastatic lesion case. The vertical axis shows the rate of reduction or increase compared with baseline (\%), and the horizontal axis shows the time after TKI treatment (years). TKI, tyrosine kinase inhibitor.
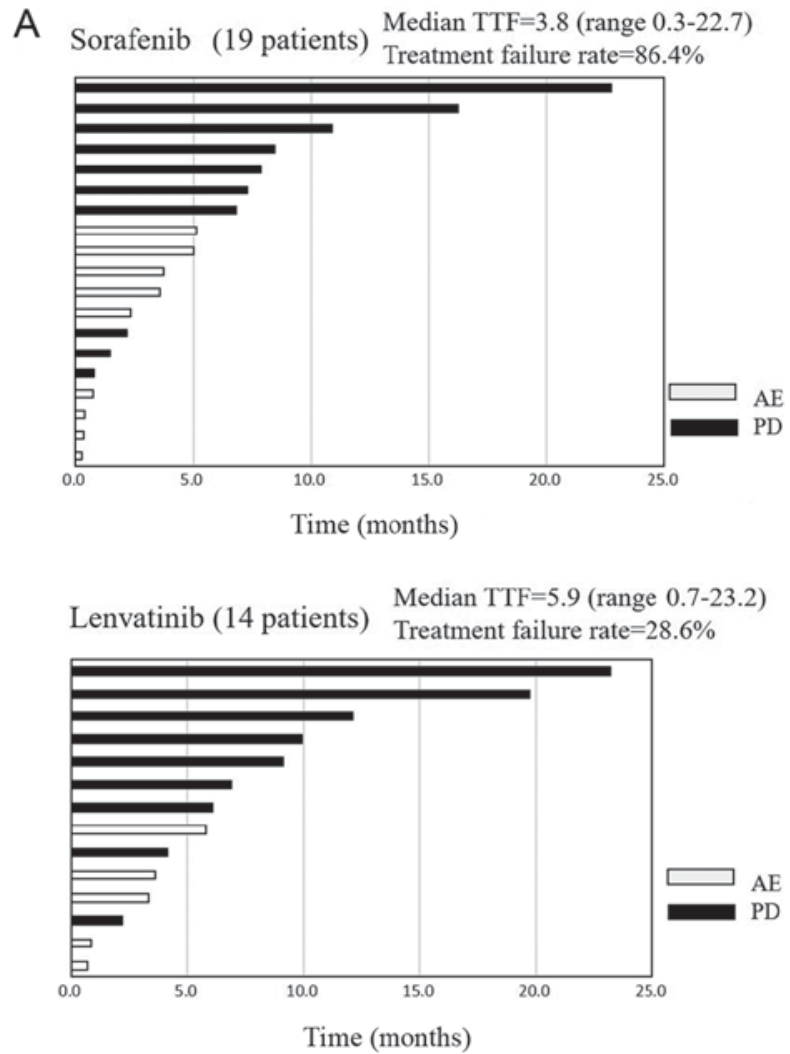

B Sorafenib (3 patients): Median duration=19.0 (range 9.0-20.9)

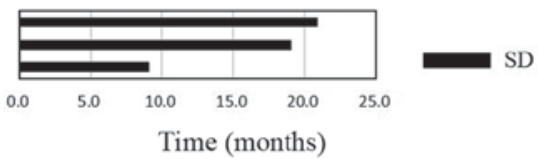

Lenvatinib (35 patients): Median duration=17.1 (range 2.0-30.3)

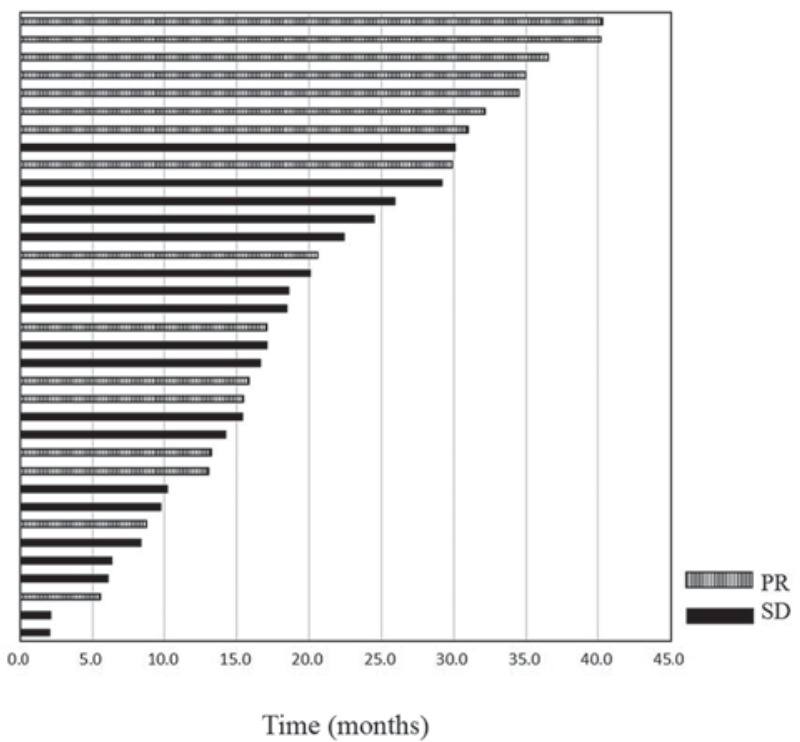

Figure 4. (A) Time to treatment failure (TTF) with sorafenib (19 patients) or lenvatinib (14 patients). The horizontal axis shows the duration (months) since initiation of TKI treatment. The black bars indicate PD and the white bars indicate AE as the reason for treatment failure. (B) Progress of ongoing treatment with sorafenib ( 3 patients) or lenvatinib (35 patients). The vertical axis shows individual patients, whereas the horizontal axis shows the duration (months) since initiation of TKI treatment. The striped bars indicate PR and the black bars indicate SD. TKI, tyrosine kinase inhibitor; AE, adverse event; PD, progressive disease; $\mathrm{SD}$, stable disease.

$14(14.3 \%)$ in the SD, TKI, and UT groups, respectively. These Tg-DT distributions were graphically demonstrated in Fig. 2.
Treatment outcomes. In total, nine patients $(39.1 \%)$ with pulmonary metastasis and seven $(29.2 \%)$ with local and lymph node recurrence exhibited PRs (Table II). Two patients 

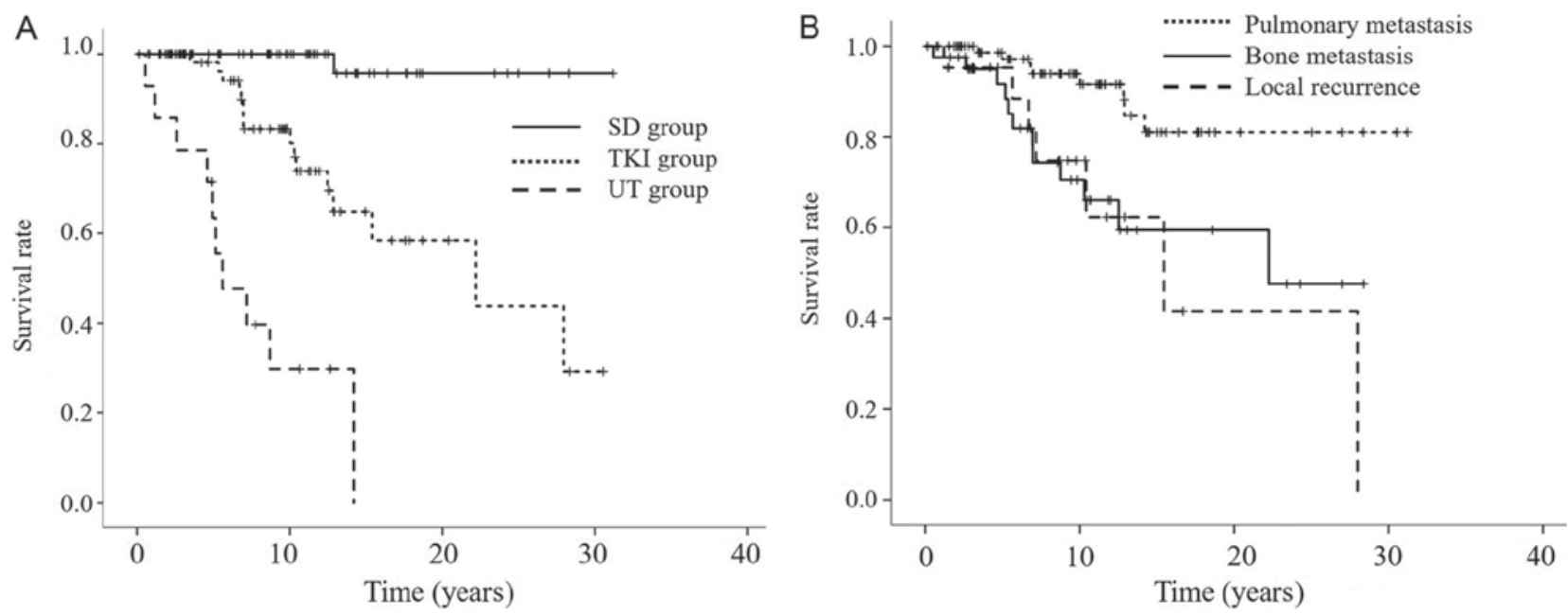

Figure 5. (A) Overall survival (OS) curves. A Kaplan-Meier estimator (SPSS software) was used to derive survival curves, which indicate the median overall survival durations as 5.6 years in the UT group and 22.2 years in the TKI group. There is a significant difference in the OS curves between the two groups so far. (B) Overall survival curves by target lesions. A Kaplan-Meier estimator (SPSS software) was used to derive survival curves, which indicate the median overall survival durations was not reached in the pulmonary metastasis, as 15.4 years in the bone metastasis and 12.9 years in the local recurrence. There is a significant difference in OS curves between the pulmonary metastasis and other two groups so far. SD, Stable disease; TKI, tyrosine kinase inhibitor; UT, untreated.

with pulmonary metastasis did not respond to TKI and died, whereas tumors shrunk in the remaining 21 cases. (Fig. 3A) In those with lymph node recurrence, three cases had quick regrowth following discontinuation of lenvatinib (Fig. 3B). Moreover, although tumor shrinkage was noted in patients with bone metastasis, no patient exhibited PR, and the rest exhibited SDs (Table II and Fig. 3C). In terms of target lesions, TKI treatment was most effective for pulmonary metastasis. (Table II and Fig. 3A-C).

The median TTF for sorafenib was 3.8 (range, 0.3-22.7) months, and the treatment failure rate was $86.4 \%$ (19 of 22 patients). Conversely, the median TTF for lenvatinib was 5.9 (range, 0.7-23.2) months, and the treatment failure rate was 14 of 49 (28.6\%). The reasons for discontinuation of sorafenib were as follows: 10 cases were PD, 9 cases were $\mathrm{AE}$ (4 cases of allergic dermatitis, 2 cases of gastrointestinal bleeding, and 1 case each of liver dysfunction, anorexia, and whole body malaise). For lenvatinib, nine cases were PD and five cases were AE (two cases of grade 5 bleeding and one case each of gastrointestinal perforation, allergic dermatitis, and anorexia). The median treatment duration for sorafenib was 5.1 (range, 0.3-22.7) months and that for lenvatinib was 14.1 (range, 0.7-40.3) months (Fig. 4A and B). Drug evaluation was performed with TTF and on-going TKI, i.e., a drug with a longer TTF period and lower failure rate was better, and clearly a drug with a larger number of on-going TKI was better. The TTF period was longer for lenvatinib, and treatment failure rate was higher for sorafenib. On-going treatment included 35 cases of lenvatinib and 3 cases of sorafenib. Lenvatinib is widely-used in clinical practice.

The median OS was 5.61 years (95\% confidence interval, 2.18-9.04) in the UT group whereas that was 22.2 years (95\% confidence interval, 8.85-35.57) in the TKI group (Fig. 5A). The OS of TKI-treated patients was better than that of UT patients, with statistically significant difference between the groups (log-rank test, $\mathrm{P}<0.001$ ).The median OS was 15.42 years (95\% confidence interval, 6.17-24.67) for bone metastasis, 22.21 years (95\% confidence interval, 10.41-NA) for local recurrence, and was not attained for pulmonary metastasis (Fig. 5B). The prognosis by target lesion was satisfactory for pulmonary metastasis, and there is statistically significant difference between the groups (log-rank test, $\mathrm{P}<0.001)$.

\section{Discussion}

Efficacy of TKI therapy by target lesion. The lesion evaluation criterion of RECIST is a tumor diameter of at least $10 \mathrm{~mm}$ on CT (19), thus only tumors of this size should be assessed to determine the initial treatment effect. In other words, in order to diagnose a PR (30\% decrease) at a tumor diameter of $10 \mathrm{~mm}$, the diameter before treatment must be at least $14.3 \mathrm{~mm}$. Therefore, in patients with pulmonary metastasis, TKI therapy is only considered in those with a maximum tumor diameter of at least $15 \mathrm{~mm}$ or alternatively $10 \mathrm{~mm}$ in patients with symptomatic lesions; hence, we established these standards so that TKI introduction timing is not too late to stop disease progression. In the TKI group, two patients with pulmonary metastasis $(10-15 \mathrm{~mm})$ were symptomatic; the remainder of metastases were $>15 \mathrm{~mm}$. We did not experience a symptomatic patient with pulmonary metastasis $<10 \mathrm{~mm}$. As shown in Figs. $3 \mathrm{~A}$ and $5 \mathrm{~B}$, the prognosis of pulmonary metastasis cases treated with TKI was the best, so such introduction criteria seemed to be successfully approved so far. If possible, surgical resection should be performed for local and lymph node recurrence including parapharyngeal metastasis (20); however, cases recurring multiple surgeries or those diagnosed as unresectable had previously undergone external irradiation or additional RAI therapy. Lamartina et al reported that the rate of CR following the first reoperation due to persistent/recurrent DTC was 53\% at the last assessment after a median of 
5 years (21). Furthermore, TKI treatment of lesions involving the common carotid artery and mediastinal large vessels should be administered with caution (22). An example of a lesion requiring particular attention with TKI treatment is parapharyngeal metastasis. Among five patients with parapharyngeal metastasis, three demonstrated syncopal attacks due to carotid sinus syndrome (23) based on the metastatic lesion, which is a serious symptom to start TKI treatment. Among patients with bone metastasis, some metastatic lesions, such as those on the ribs, are resectable, but bone load and vertebral body bone metastasis may cause pathologic fractures and spinal cord paralysis. Consequently, early initiation of TKI treatment will prolong the quality of life. In the case of spinal metastasis, if a risk of spinal paralysis due to the withdrawal of thyroid hormone is observed while preparing for RAI therapy, it should be preceded by decompression surgery and external irradiation (24), and then TKI treatment should be initiated. The therapeutic effects of TKI are shown in Table II and Fig. 3. The tumor diameter of lung metastasis as the target lesion was relatively small, and the therapeutic effect was most satisfactory. In contrast, bone metastasis with a large tumor diameter had a poorer therapeutic effect. Other lesions showed intermediate results (Table II). Both tumor size prior to TKI treatment and metastasis site are believed to affect the therapeutic effect.

$T g$-DT as the indicator for disease progression. On the basis of a previous report that Tg-DT reflects the prognosis of DTC (9), we examined the prognostic value of this variable following the introduction of TKI treatment. The incidence of $\mathrm{TgAb}$ positivity was statistically high both in the TKI and UT groups (Table II), but those Tg-DTs were not informative. Although there were 64 of 147 informative cases (43.5\%), the calculated Tg-DT was not statistically significant between the SD group and the TKI and UT groups (Fig. 2 and Table II). Tg levels either decreased or insignificantly increased in several patients in the SD group, and RAI therapy or external irradiation was successful; such findings reflect a lack of disease progression, making TKI treatment unnecessary. In other words, as Tg levels do not increase with treatment success (17), it is important to measure Tg during both TKI treatment and postoperative SD. Both reversed $\mathrm{TgAb}$ positivity (25) and increased $\mathrm{Tg}$ levels (26) indicate disease progression; however, Tg-DT did not accurately reflect tumor growth when compared with imaging assessments. Although Tg-DT is not an indicator of disease progression in many cases, the value was $<1$ year in six patients in the SD group and eight patients in the TKI group. Although Tg-DT is useful for the detection of recurrence and distant metastases, it was not an indicator of disease progression in cases with existing distant metastases. The frequency of $\operatorname{Tg} \mathrm{Ab}$ increased with disease progression, and the rate of increase of $\mathrm{Tg}$ level became low for further disease progression.

TKI treatment to prolong survival of DTC with distant metastasis. No increase in Tg levels or the number of metastatic lesions was observed in the SD group; therefore, TKI treatment was not required. Although it is obvious, there have been no previous reports demonstrating that the OS of patients with distant metastasis at the initial diagnosis for DTC is worse than that of patients recognized with distant metastasis postoperatively (Fig. 4C). Moreover, 10 of 14 patients (71.4\%) in the UT group died, and the median OS in the TKI group was 22.2 years. This finding is particularly significant due to the extended SD period of this retrospective study. Although the study population of UT group was limited, the OS of the TKI and UT groups was significantly different, it is important to further improve survival by starting TKI treatment at appropriate time. The treatment outcomes of TKI included treatment failure due to $\mathrm{AE}$, three cases of regrowth phenomenon, and three cases of AE death. Because of PD in 10/22 (45.5\%) sorafenib cases and 5/49 (10.2\%) lenvatinib cases, they are considered non-responders (Fig. 4). Other cases were responders, and tumor shrinkage was observed as shown in Fig. 3. Outcomes may be further improved by AE management. The prevention of toxicities is necessary to allow patients to remain on treatment as long as possible without dose or schedule modifications (27).

However, the existence of AEs cannot be ignored (11), as two patients with recurrent tumors involving large vessels experienced fatal bleeding following TKI therapy. According to the Common Terminology Criteria for Adverse Events, version 3.0, dose reduction or treatment withdrawal should be initiated in cases of renal dysfunction or skin fistula. Meanwhile, three patients experienced sudden tumor regrowth following drug withdrawal, leading to decreased treatment outcomes. Despite the satisfactory DCR, it must be noted that no CRs were achieved following TKI therapy. Thus, it may be necessary to continue TKI therapy as long as possible. Because TKI treatment is the final option, treatment failure resulted in death. As more cases of long-term TKI treatment will be observed in the future, we believe that proper dose reduction and drug withdrawal guidelines are necessary; in absence of these, more patients will die from tumor regrowth following discontinuation of TKIs. Therefore, it is hard to predict whether TKI therapy, which is expensive and has various AEs, contributes to long-term improvement in the treatment outcomes for distant metastasis from DTC.

Distant metastases from DTCs comprise a heterogeneous group of thyroid cancers with diverse histology, disease progression, and genomic alterations. Due to the limited treatment options, the OS is usually poor among patients with aggressive metastatic disease (28). In this study, we report a high $\mathrm{Tg}$-Ab-positive rate in patients with disease progression; however, Tg-DT was not informative for PD diagnosis. The OS was poor among patients with distant metastasis at the initial diagnosis for DTC. TKI treatment does improve OS in distant metastases DTCs, especially in patients with pulmonary metastasis. Management of this unique group of patients will improve as we gain a better understanding of the optimal timing of TKI therapy administration.

\section{Acknowledgements}

The authors thank Dr Akira Yoshida (Center Clinic of Kanagawa Health Service Association) and Dr Yoshio Kure (Director of Kure Clinic) for referring several patients to our hospital. An abstract (poster 1139) covering a part of this article was presented at the 20th European congress of Endocrinology, May 19-22, Barcelona, Spain in 2018. 


\section{Funding}

No funding was received.

\section{Availability of data and materials}

The datasets used and/or analyzed during the current study are available from the corresponding author on reasonable request.

\section{Authors' contributions}

HI and HY designed the study. HT and RS checked analysis and interpretation data, especially statistical analysis. NS, HN, SH, ST and KM contributed by performing the surgery and caring for the patients. NS, HN, SH contributed to analysis and interpretation data, particularly drug efficacy and clinical assessment. ST and KM contributed to data acquisition. All authors read and approved the final manuscript.

\section{Ethics approval and consent to participate}

The chemotherapy committee of Kanagawa Cancer Center (Yokohama, Kanagawa, Japan) approved this regimen of lenvatinib or sorafenib for use in patients with DTC. The cancer board of the hospital also approved these TKI treatment, including surgery, for patients with DTC. The study was approved by the Institutional Review Board of Kanagawa Cancer Center.

\section{Patient consent for publication}

All patients provided a comprehensive consent form stating that personal data may be used for academic presentation or paper presentation while ensuring complete anonymity prior to receiving treatment.

\section{Competing interests}

The authors declare that they have no competing interests.

\section{Authors' information}

$\mathrm{HI}$ is an endocrine surgeon working at the Kanagawa Cancer Center and has an extensive experience of several surgeries for advanced thyroid cancer as well as ATC treatment.

\section{References}

1. Carhill AA, Litofsky DR, Ross DS, Jonklaas J, Cooper DS, Brierley JD, Ladenson PW, Ain KB, Fein HG, Haugen BR, et al: Long-term outcomes following therapy in differentiated thyroid carcinoma: NTCTCS registry analysis 1987-2012. J Clin Endocrinol Metab 100: 3270-3279, 2015.

2. Scheffel RS, Zanella AB, Dora JM and Maia AL: Timing of radioactive iodine administration does not influence outcomes in patients with differentiated thyroid carcinoma. Thyroid 26: 1623-1629, 2016

3. Kwong N, Marqusee E, Gordon MS, Larsen PR, Garber JR, Kim MI and Alexander EK: Long-term, treatment-free survival in select patients with distant metastatic papillary thyroid cancer. Endocr Connect 3: 207-214, 2014.

4. Brink JS, van Heerden JA, McIver B, Salomao DR, Farley DR, Grant CS, Thompson GB, Zimmerman D and Hay ID: Papillary thyroid cancer with pulmonary metastases in children: Long-term prognosis. Surgery 128: 881-887, 2000.
5. Goffredo P, Sosa JA and Roman SA: Differentiated thyroid cancer presenting with distant metastases: A population analysis over two decades. World J Surg 37: 1599-1605, 2013.

6. Haugen BR: 2015 American thyroid association management guidelines for adult patients with thyroid nodules and differentiated thyroid cancer: What is new and what has changed? Cancer 123: 372-381, 2017.

7. Nixon IJ, Wang LY, Migliacci JC, Eskander A, Campbell MJ, Aniss A, Morris L, Vaisman F, Corbo R, Momesso D, et al: An international multi-institutional validation of age 55 years as a cutoff for risk stratification in the AJCC/UICC staging system for well-differentiated thyroid cancer. Thyroid 26: 373-380, 2016.

8. Machiels JP, Henry S, Zanetta S, Kaminsky MC, Michoux N, Rommel D, Schmitz S, Bompas E, Dillies AF, Faivre S, et al: Phase II study of sunitinib in recurrent or metastatic squamous cell carcinoma of the head and neck: GORTEC 2006-01. J Clin Oncol 28: 21-28, 2010.

9. Schlumberger M, Tahara M and Wirth LJ: Lenvatinib in radioiodine-refractory thyroid cancer. N Engl J Med 372: 1868, 2015.

10. Schlumberger M, Tahara M, Wirth LJ, Robinson B, Brose MS, Elisei R, Habra MA, Newbold K, Shah MH, Hoff AO, et al: Lenvatinib versus placebo in radioiodine-refractory thyroid cancer. N Engl J Med 372: 621-630, 2015.

11. Brose MS, Nutting CM, Jarzab B, Elisei R, Siena S, Bastholt L, de la Fouchardiere C, Pacini F, Paschke R, Shong YK, et al: Sorafenib in radioactive iodine-refractory, locally advanced or metastatic differentiated thyroid cancer: A randomised, double-blind, phase 3 trial. Lancet 384: 319-328, 2014.

12. Ito Y, Suzuki S, Ito K, Imai T, Okamoto T, Kitano H, Sugitani I, Sugino K, Tsutsui H, Hara H, et al: Tyrosine-kinase inhibitors to treat radioiodine-refracted, metastatic, or recurred and progressive differentiated thyroid carcinoma (Review). Endocr J 63: 597-602, 2016.

13. Perrier ND, Brierley JD and Tuttle RM: Differentiated and anaplastic thyroid carcinoma: Major changes in the American Joint Committee on Cancer eighth edition cancer staging manual. CA Cancer J Clin 68: 55-63, 2018.

14. Gül G, Sendur MA, Aksoy S, Sever AR and Altundag K: A comprehensive review of denosumab for bone metastasis in patients with solid tumors. Curr Med Res Opin 32: 133-145, 2016.

15. Eisenhauer EA, Therasse P, Bogaerts J, Schwartz LH, Sargent D, Ford R, Dancey J, Arbuck S, Gwyther S, Mooney M, et al: New response evaluation criteria in solid tumours: Revised RECIST guideline (version 1.1). Eur J Cancer 45: 228-247, 2009.

16. Miyauchi A, Kudo T, Miya A, Kobayashi K, Ito Y, Takamura Y, Higashiyama T, Fukushima M, Kihara M, Inoue H, et al: Prognostic impact of serum thyroglobulin doubling-time under thyrotropin suppression in patients with papillary thyroid carcinoma who underwent total thyroidectomy. Thyroid 21: 707-716, 2011.

17. Werner RA, Lückerath K, Schmid JS, Higuchi T, Kreissl MC, Grelle I, Reiners C, Buck AK and Lapa C: Thyroglobulin fluctuations in patients with iodine-refractory differentiated thyroid carcinoma on lenvatinib treatment-initial experience. Sci Rep 6: 28081, 2016.

18. Kanda Y: Investigation of the freely available easy-to-use software 'EZR' for medical statistics. Bone Marrow Transplant 48: 452-458, 2013

19. Levine ZH, Borchardt BR, Brandenburg NJ, Clark CW, Muralikrishnan B, Shakarji CM, Chen JJ and Siegel EL: RECIST versus volume measurement in medical CT using ellipsoids of known size. Opt Express 18: 8151-8159, 2010.

20. Giordano L, Pilolli F, Toma S and Bussi M: Parapharyngeal metastases from thyroid cancer: Surgical management of two cases with minimally-invasive video-assisted technique. Acta Otorhinolaryngol Ital 35: 289-292, 2015.

21. Lamartina L, Borget I, Mirghani H, Al Ghuzlan A, Berdelou A, Bidault F, Deandreis D, Baudin E, Travagli JP, Schlumberger M, et al: Surgery for neck recurrence of differentiated thyroid cancer: Outcomes and risk factors. J Clin Endocrinol Metab 102: 1020-1031, 2017.

22. Hui EP, Ma BB, King AD, Mo F, Chan SL, Kam MK, Loong HH, Ahuja AT, Zee BC and Chan AT: Hemorrhagic complications in a phase II study of sunitinib in patients of nasopharyngeal carcinoma who has previously received high-dose radiation. Ann Oncol 22: 1280-1287, 2011.

23. Epstein SS and Shaw HJ: Metastatic cancer of the larynx as a cause of carotid-sinus syndrome. Cancer 10: 933-937, 1957.

24. Ramadan S, Ugas MA, Berwick RJ, Notay M, Cho H, Jerjes W and Giannoudis PV: Spinal metastasis in thyroid cancer. Head Neck Oncol 4: 39, 2012. 
25. Morbelli S, Ferrarazzo G, Pomposelli E, Pupo F, Pesce G, Calamia I, Fiz F, Clapasson A, Bauckneht M, Minuto M, et al: Relationship between circulating anti-thyroglobulin antibodies $(\mathrm{Tg} \mathrm{Ab})$ and tumor metabolism in patients with differentiated thyroid cancer (DTC): Prognostic implications. J Endocrinol Invest 40: 417-424, 2017.

26. Neshandar Asli I, Siahkali AS, Shafie B, Javadi H and Assadi M: Prognostic value of basal serum thyroglobulin levels, but not basal antithyroglobulin antibody (TgAb) levels, in patients with differentiated thyroid cancer. Mol Imaging Radionucl Ther 23: 54-59, 2014.
27. Resteghini C, Cavalieri S, Galbiati D, Granata R, Alfieri S, Bergamini C, Bossi P, Licitra L and Locati LD: Management of tyrosine kinase inhibitors (TKI) side effects in differentiated and medullary thyroid cancer patients. Best Pract Res Clin Endocrinol Metab 31: 349-361, 2017.

28. Yeung KT and Cohen EE: Lenvatinib in advanced, radioactive iodine-refractory, differentiated thyroid carcinoma. Clin Cancer Res 21: 5420-5426, 2015. 